\title{
Determination of Paeonol in Mingmu Dihuang Pill by Capillary Electrophoresis
}

\author{
Hai-Xing Liu, ${ }^{1, a}$, Ting-Ting Huang ${ }^{1, b}$, Lin-Tong Wang ${ }^{1, c}$, Yin-Ping Zhang ${ }^{1, d}$ and \\ Xia Wang ${ }^{1, \mathrm{e}}$
}

${ }^{1}$ Chemistry \& Chemical and Environmental Engineering College, Weifang University, Weifang 261061, P.R. China

ahaixingliu@tom.com, b2_haixingliu@tom.com, ${ }^{\mathrm{c}} 3$ _haixingliu@tom.com, ${ }^{\mathrm{d}} 5$ _haixingliu@tom.com, e4_haixingliu@tom.com,

Keywords: capillary electrophoresis, paeonol, Mingmu Dihuang pill

Abstract: The High Performance Capillary Electrophoresis (HPCE) method was established for thr determination the paeonol content in Mingmu Dihuang pill. 40mmol/L borax solution buffer was used for the electrophoresis analysis. Measured paeonol content in Mingmu Dihuang pill was 1.698 $\mathrm{mg} / \mathrm{g}(\mathrm{RSD}=10.8 \%)(\mathrm{n}=4)$.

\section{Introduction}

Mingmu Dihuang Pill is composed of medlar, chrysanthemum, Rehmannia glutinosa, cornel, moutan bark, yam, fuling, alisma, Angelica sinensis, Radix paeoniae alba etc. traditional Chinese medicine preparation. It has the nutrition of kidney, maintenance of the liver, bright eye effect.It is used for liver kidney weakness, the eye dry, afraid of light, blurred vision, eyes tear in the wind at the clinical [1]. Cheng et al [2] established a RP-HPLC method to determine the content of loganin in Mingmu Dihuang pill. The analysis was executed on a Kromasil $\mathrm{C}_{18}$ column $(4.6 \mathrm{~mm} \times 250 \mathrm{~mm}$, $5 \mu \mathrm{m})$ with tetrahydrofuran-acetonitrile-methanol (1:8:4)-0.05\% phosphoric acid (8:92) as mobile phase at a flow rate of $1.0 \mathrm{~mL} \cdot \mathrm{min}^{-1}$. The detection wavelength was at $236 \mathrm{~nm}$. An HPLC method was set up for the quantitative analysis of Paeoniflorin in the Mingmu Dihuang Pill by Cheng [3]. The separation was performed on Hypersil $\mathrm{C}_{18}(4.6 \mathrm{~mm} \times 250 \mathrm{~mm}, 5 \mu \mathrm{m})$ column with a mixture of acetonitrile-0.05 $\mathrm{mol} / \mathrm{L}$ potassium dihydrogen phosphate $(12: 88)$ as mobile phase. Microwave-assisted extraction (MAE) followed by headspace solid-phase microextraction (HS-SPME) gas chromatography-mass spectrometry was developed for rapid determination of paeonol in four traditional Chinese medicinal preparations (TCMPs) including Liuwen Dihuang pills, Maiwei Dihuang pills, Guifu Dihuang pills, and Zhibai Dihuang pills by Ye et al [4]. The optimal MAE conditions obtained were microwave power of $540 \mathrm{~W}$ and irradiation time of $4 \mathrm{~min}$, and HS-SPME optimal conditions were fiber coating of $65 \mu \mathrm{m}$ PDMS/DVB, extraction temperature of $70^{\circ} \mathrm{C}$, extraction time of $10 \mathrm{~min}$, stirring rate of $1100 \mathrm{rpm}$, and $\mathrm{NaCl}$ concentration $30 \%$. The optimized method provided satisfactory precision, good linearity and recovery. Xiao et al [5] improved a new method for the determination of ursolic acid in Mingmu Dihuang pill. The content of ursolic acid was obtained by HPLC and TLCS. The HPLC is simpler, more rapid and accurate, and can better control the quality of Mingmu Dihuang pill than the TLCS. Chen et al [6] determined the content of catalpol in Mingmu Dihuang pill. Cheng et al [7] established a method for the determination of paeonol in Mingmu Dihuang pills (concentrated pills) by RP-HPLC. The analysis was executed on a Agilent Zorbax SB-C 18 column $(4.6 \mathrm{~mm} \times 250 \mathrm{~mm}, 5 \mu \mathrm{m})$ with methanol-water (48:52) as mobile phase. The detection wavelength was at $274 \mathrm{~nm}$. In this paper, the paeonol content in Mingmu Dihuang pill was determined by High Performance Capillary Electrophoresis. 


\section{Experimental section}

\section{Instruments and Reagents}

Experimental instruments: CL-1030-type high performance capillary electrophoresis (Beijing Cailu Scientific Instrument Co., Ltd.); HW2000-type chromatography workstation (Nanjing Qianpu Software Ltd.); Capillary (75 $\mu \mathrm{m}$ inner diameter, $60 \mathrm{~cm}$ overall length, $52 \mathrm{~cm}$ effective length) from Hebei Yongnian Ruifeng Chromatographic Devices Co., Ltd.).

Paeonol (Chinese Drugs and Biological Products); Mingmu Dihuang pill (Neimenggu tianqi zhongmeng pharmaceutical Co., Ltd.); Other reagents used in the experiments were all analytical grade; Double-distilled water was used.

\section{Experimental Methods}

Before the start of the experiment, capillary was successively washed with $1 \mathrm{~mol} \cdot \mathrm{L}^{-1}$ hydrochloric acid solution, double-distilled water, $1 \mathrm{~mol} \cdot \mathrm{L}^{-1}$ sodium hydroxide solution, double-distilled water, buffer solution, each for $8 \mathrm{~min}$. After three times running, capillary was cleaned again using the above method.

Measurements were carded out at $20 \mathrm{kV}$ voltage and $14{ }^{\circ} \mathrm{C}$ experimental temperature. UV detection wavelength was $270 \mathrm{~nm}$. Injection time was $10 \mathrm{~s}$ ( $7.5 \mathrm{~cm}$ height difference).

\section{Sample Preparation}

Mingmu Dihuang pill sample solution: Mingmu Dihuang pill powder was accurately weighed $1.9155 \mathrm{~g}$, added $30 \mathrm{~mL}$ water with $25 \%$ ethanol, cold soak time of $24 \mathrm{~h}$, filtered, washed and set the volume to $50 \mathrm{~mL}$ that was the Mingmu Dihuang pill sample solution.

Paeonol standard solution: Paeonol was accurately weighed $0.0012 \mathrm{~g}$, added $3 \mathrm{~mL}$ water with $25 \%$ ethanol.

\section{Results and Discussion}

\section{Selection electrophoresis conditions}

Based on past experiment experience, we chose $40 \mathrm{mmol} / \mathrm{L}$ borax solution as a running buffer solution.

According to the literature, paeonol maximum absorption wavelength was at $274 \mathrm{~nm}$, so we chose the $270 \mathrm{~nm}$ detection wavelength.

\section{Quantitative analysis}

\section{Standard curve}

First, paeonol standard solution that the concentration were $0.4,0.2,0.1,0.05,0.025,0.0125$, $0.00625,0.003125 \mathrm{mg} / \mathrm{mL}$ was prepared. Each standard solution was run for three times under the above electrophoresis conditions and the results averaged. The chromatogram of paeonol standard solution was showed in Figure 1. Taking concentration as the abscissa and peak area as the ordinate, the standard curve was drew. Linear regression equation of paeonol (peak area: $\mathrm{y} \mu \mathrm{V} \bullet \mathrm{s}$, density: $\mathrm{x}$ $\mathrm{mg} / \mathrm{mL})$ and the linear range was as follows: $\mathrm{y}=-3147.5+420341 \mathrm{x}(\mathrm{r}=0.998), 0.003-0.4 \mathrm{mg} / \mathrm{mL}$. 


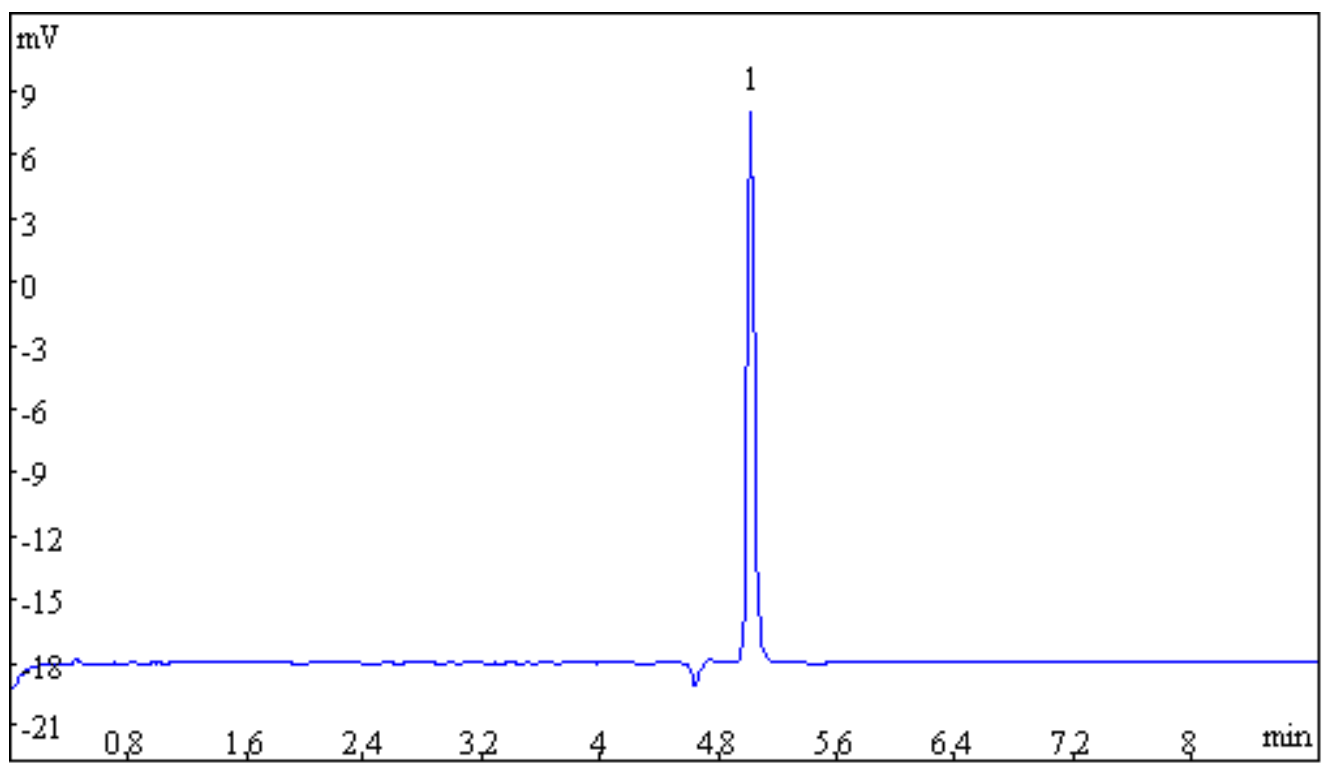

Fig.1 Electrophorogram of paeonol standard solution 1-paeonol

\section{Precision test}

Paeonol standard solution precisely drew and continuously injected for six times under electrophoretic separation conditions, the RSD of paeonol peak area was 3.3\%, indicating good precision.

\section{Determination of sample content}

Under selected electrophoresis conditions, Mingmu Dihuang pill sample solution was run. Separation chromatogram of the Mingmu Dihuang pill sample solution was showed in Figure 2. Measured paeonol content in Mingmu Dihuang pill was $1.698 \mathrm{mg} / \mathrm{g}(\mathrm{RSD}=10.8 \%)(\mathrm{n}=4)$.

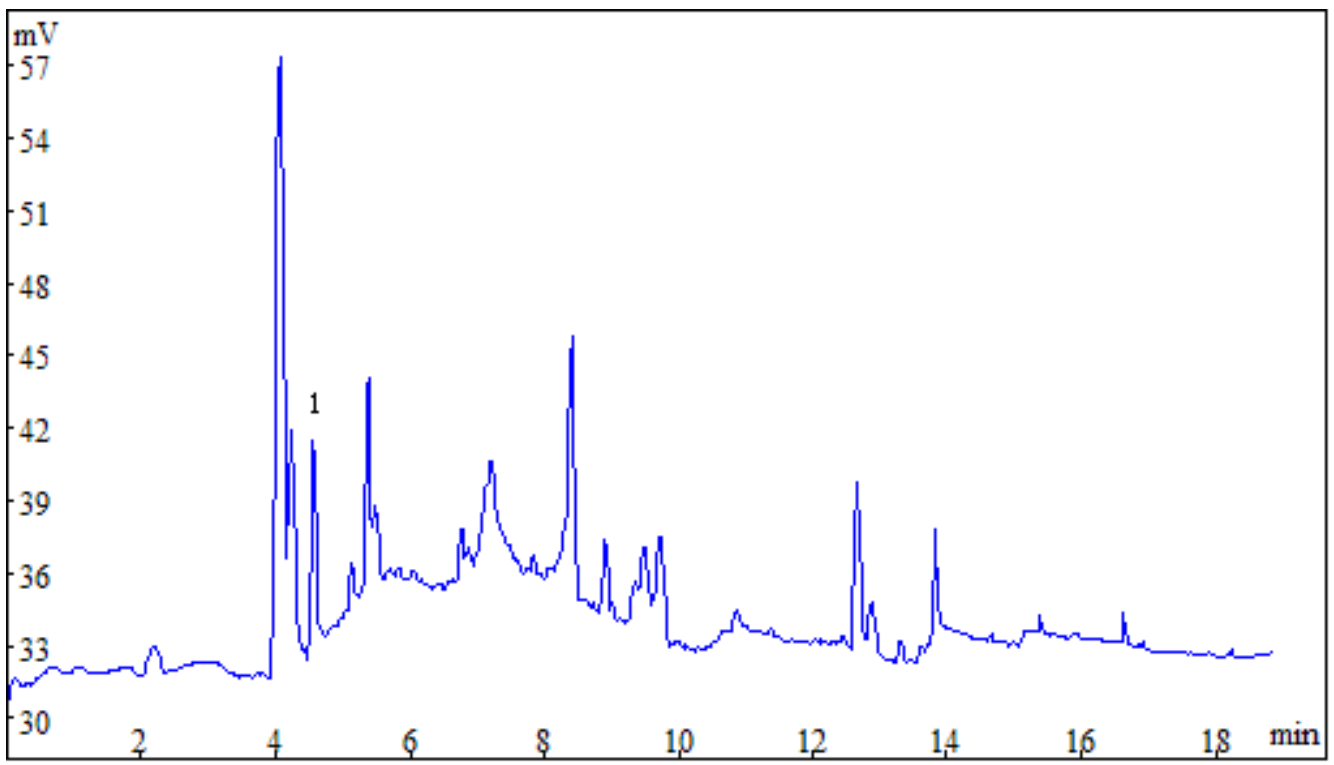

Fig.2 Electrophorogram of Mingmu Dihuang pill sample solution 1-paeonol 


\section{Recovery}

After determination for four times, the recovery of paeonol in Mingmu Dihuang pill sample was in the range of $77.8 \%-109.4 \%(n=4)$.

\section{Acknowledgments}

This study were supported by the Natural Science Foundation of Shandong Province (No. ZR2010BL025), Open Project of State Key Laboratory of Supramolecular Structure and Materials (No. sklssm201323)(Jilin University), State Key Laboratory of Inorganic Synthesis and Preparative Chemistry (No. 2011-13)(Jilin University).

\section{References}

[1] Xiao-guang Xu, Ren-gang Cheng, Xiao-lei Yu, Qilu pharmaceutical (In Chinese), 2004, 23(6), 229-230

[2] Qiao-yuan Cheng, Chun-mei Tan, Chinese Journal of Experimental Traditional Medical Formulae (In Chinese), 2012, 18(7), 68-70

[3] He-li Cheng, Anhui Medical and Pharmaceutical Journal (In Chinese), 2008, 12(4), 314-315

[4] Qing Ye, Da-gui Zheng, Zong-bao Chen, Journal of Analytical Chemistry (In Chinese), 2011, 66(3), 285-289

[5] Pei-yun Xiao, Yong-shou Yang, Jia-qiang Xu, Journal of Dali University (In Chinese), 2010, $9(12), 4-6$

[6] Jing Chen, Rong Tang, Practical traditional Chinese and western medicine clinical (In Chinese), 2004, 4(2), 66

[7] Qiao-yuan Cheng, Bi-lian Chen, Herald of Medicine (In Chinese), 2012, 31(5), 630-632 